\title{
PROCEDIMIENTOS DE MUESTREO REPRESENTATIVO DE LAS PERSONAS SIN HOGAR (PSH) Avance de un estudio descriptivo en Madrid *
}

\author{
M. MUÑOZ, C. VÁZQUEZ, J.J. VÁZQUEZ, A.B. GUISADO y M. CRESPO \\ Universidad Complutense de Madrid
}

PALABRAS CLAVE ADICIONALES

Sin Techo, Exclusión social, Epidemiología,

Estudio Psicosocial, Metodología, Noche S.

\section{ADDITIONAL KEYWORDS}

Homeless, Homelesness, Epidemiology, Phychosocial Study, Methodology, S Night.

RESUMEN. El presente trabajo se enmarca en el Proyecto Sin Hogar (Muñoz y Vázquez, 1996). Se exponen las estrategias de muestreo utilizadas que pueden servir para ofrecer datos no sesgados sobre las Personas Sin Hogar (PSH) o sobre otros grupos de difícil localización. Junto a las estrategias de muestreo utilizadas para conseguir la máxima representatividad de la muestra, se incluyen los principales datos descriptivos sociodemográficos obtenidos con este procedimiento. La estrategia de muestreo representativo incluye la realización de un listado exhaustivo de centros para PSH, una Noche S (muestreo en calles en una noche determinada) para determinar la frecuencia de uso de recursos por parte de las PSH y, finalmente, un sistema de muestreo en estos recursos que asegure la representatividad en función del día de la semana, tipo de centro en que se realizó el contacto y tamaño de cada centro. Los resultados mostrados en este estudio incluyen estadísticos descriptivos y porcentajes de las principales variables descriptivas sociodemográficas y de actividad económica. Se incluyen los datos directos y ponderados y se analizan las principales diferencias que se producen como efecto de la ponderación estadística efectuada.

ABSTRACT. The present paper is framed within the Homeless Project (Muñoz y Vázquez, 1996). The main aim of this project is the study of economic, psychosocial and health factors affecting homeless people in Madrid. In this study we show the sampling strategies used to obtain a representative sample of data on homelessness. The sampling strategies included are very innovative in the Spanish context. Besides the sampling method characteristics, we include the main descriptive results on sociodemographic variables.The sampling strategy began by collecting an exhaustive

E-mail: mmunoz@psi.ucm.es

- Estudio cofinanciado por el proyecto SEC96-1008-95 (Plan Nacional de I+D, CICYT) y el Proyecto Inclusión (Plan nacional I+D+I, 2000/03).

Revista Internacional de Sociología (RIS)

Tercera Época, $\mathrm{n}^{\circ}$ 32, Mayo-Agosto, 2002, pp. 155-180. 
RIS

REVISTA INTERNACIONAL DE SOCIOLOGÍA

No 32, Mayo-Agosto, 2002

M. MUÑOZ, C. VÁZQUEZ, JJ. VÁQUEZ, A.B. GUISADO Y M. CRESPO

list of centers for homeless people and a so-called S-Night (i.e., street sampling in a given night) to determine the frequency of use of resources for homeless people. To avoid sampling biases, the sampling methodology also considered the influence of both the size and center type (e.g., shelter vs. soup kitchen) and the day of the week in which the contact was done. The data obtained were statistically weighted as a function of differences betwen the estimated and the empiric sample size and as a function as well of the frequency of use of services. Results show descriptive statistics and percentages on the more important sociodemographic variables included in the study. Weighted and unweighted data and their differences are analyzed.

\section{INTRODUCCIÓN}

Aunque el fenómeno de las Personas Sin Hogar (PSH) es un tema de constante actualidad en los medios de comunicación y la visibilidad del mismo es muy alta, el conocimiento acumulado sobre las condiciones que llevan a las personas a estar en esa situación y las características de las mismas no ha seguido un desarrollo paralelo. De hecho en la Unión Europea, incluida España, ni siquiera se cuenta con una definición común oficial de Persona Sin Hogar llegando, de este modo, al extremo máximo de la exclusión social al ser en buena medida ignorado el problema por las instituciones implicadas. En consecuencia, tampoco se conocen adecuadamente las cifras exactas de incidencia y prevalencia del problema y mucho menos se identifican las características sociodemográficas, psicosociales y de salud que afectan a estas personas. De este modo la planificación de políticas preventivas y de intervención se realiza de forma parcial, con desconocimiento de la magnitud del problema o no se realiza en absoluto.

Ateniéndonos a una de las definiciones más extendidas, como es la de FEANTSA (Federación Europea de Asociaciones que trabajan con Personas Sin Hogar) (Avramov, 1995; Daly, 1994), PSH:“... es aquella persona incapaz de acceder y mantener un alojamiento personal adecuado por sus propios medios, $\mathrm{y}$ personas incapaces de mantener alojamiento con la ayuda de los Servicios Sociales". Las investigaciones realizadas sobre las PSH ponen de manifiesto que el número de individuos en esta situación viene incrementándose en las sociedades industrializadas durante los últimos años. En nuestro país, estadísticas basadas en el número de usuarios de servicios para PSH y personas con necesidades de vivienda, sugieren que, sobre una población de unos 40 millones de habitantes, alrededor de 160.000 personas se encuentran en esa situación (Avramov, 1995). De forma más estricta, si se cuentan exclusivamente aquellas personas que o bien duermen literalmente en la calle o en albergues para Personas Sin Hogar, podrían ser alrededor de 11.000 las que se encuentren en esta situación. En lo referente a la ciudad de Madrid, estimaciones basadas en trabajos de campo o estadísticas sobre recursos específicos, ofrecen datos que oscilan entre 1.500 y 3.000 personas en esta situación (Ayuntamiento de Madrid, 1986; Infante et al., 1990; Grupo 5, 1994; Cabrera, 1998, Cáritas Española, 1997). 
Sin embargo, todos estos datos resultan parciales, incompletos o sesgados. En primer lugar, las estimaciones basadas en el uso de recursos ofrecen una visión parcial del problema pues no tienen en cuenta a aquellas PSH que no utilizan los mismos. Por lo tanto se requiere una estrategia que considere de algún modo la contabilización de estas personas. En segundo lugar, el muestreo de recursos ha de ser exhaustivo dado que existe una diversidad de tipos de recursos a los que estas personas acuden y que, frecuentemente, no son revisados por los autores que, generalmente, se centran en uno o dos centros para realizar los muestreos (comedores, albergues, estaciones de metro abiertas en períodos de emergencia, roperos, etc.). En tercer lugar, la estrategia de muestreo debe evitar o controlar las duplicaciones, dado que la mayoría de las PSH utilizan simultáneamente distintos tipos de recursos. Por último, un muestreo verdaderamente representativo debe considerar la frecuencia de uso de cada usuario para cada recurso; en caso contrario la muestra siempre representará en exceso a aquellas personas que utilizan más frecuentemente los recursos mientras que los que los utilizan en menor medida siempre quedaran infrarrepresentados. La importancia de desarrollar muestreos de las PSH, que representen proporcionalmente a todos los servicios e incluyan, igualmente, a aquellas que no utilizan servicios ha sido puesta de manifiesto por diferentes autores (Rossi, 1989; Koegel et al., 1996; Mapsat, 1995), quienes han sugerido diferentes estrategias de muestreo que incluyen muestreos multicentro, multiservicio y multidia y muestreos en diferentes lugares de concentración de PSH. De este modo, a partir de los resultados obtenidos en estudios llevados a cabo en diferentes puntos de EE.UU. - utilizando estrategias de conteo que incluyen PSH no usuarias de servicios- Burt (1993) estimó que una muestra que incluyese albergues, comedores sociales y a las PSH visibles en las calles cubriría, aproximadamente, al $70 \%-80 \%$ de todas las PSH de las zonas urbanas de Estados Unidos. Sin embargo, de forma paralela, datos del Distritc of Columbia Metropolitan Area Drug (DC MADS) Homelessness Study (Dennis, 1993) - un ambicioso trabajo que utilizaba diferentes técnicas de muestreo señalaban que, al menos en Washington D.C., el 93\% de las PSH se encontrarían incluidas en un muestreo llevado a cabo únicamente en albergues y comedores sociales, alcanzándose cifras superiores al $94 \%$ si se muestreaba además entre aquellas PSH fácilmente visibles en las calles. Para identificar la proporción de PSH que quedan fuera de los muestreos multicentro se han utilizado las estrategias de "noches de muestreo" (S-night) (Wrigth y Devine, 1992; Edin, 1992; Hopper, 1992; Cousineau y Ward, 1992; Stark, 1992; Devine y Wright, 1992; Martin; 1992). Igualmente se han elaborado complejos sistemas de pesos estadísticos para corregir las desviaciones ocurridas por los distintos niveles de utilización de los servicios de cada persona (Koegel y Burnam, 1992; Marpsat y Firdiau, 1998; Burt, 1992).

En España, no utilizar estrategias de muestreo adecuadas ha dificultado la obtención de una estimación correcta de las características de las PSH. Los 
muestreos sobre población de PSH en España se han realizado, generalmente en centros de atención específicos, perdiéndose así toda representatividad de la muestra más allá del centro, o tipo de centros, escogidos. Aunque en los últimos años se han llevado a cabo algunos trabajos con estrategias de muestreo multicentro y multiservicio (Muñoz, Vázquez y Cruzado, 1995; Cabrera, 1998), no han sido absolutamente rigurosos en la aplicación del azar y la proporcionalidad. En este sentido, no se han realizado Noches $\mathrm{S}$ en ninguna ciudad española y no se han aplicado coeficientes de ponderación en función de la probabilidad de uso de los servicios.

El presente trabajo, se enmarca en una serie de proyectos que vienen desarrollándose por los autores desde el año 1992 y que engloban diferentes líneas de investigación cuantitativa y cualitativa sobre la situación y características que afectan a las Personas Sin Hogar en Madrid y su comparación transcultural con otras ciudades europeas y norteamericanas (Muñoz, Vázquez y Cruzado, 1995; Vázquez, Muñoz y Sanz, 1997; Muñoz, Vázquez, Koegel, Sanz y Burnam, 1998; Muñoz, Vázquez, Bermejo y Vázquez, 1999; Leonori et al., 2000; Muñoz, Koegel, Vázquez, Sanz y Burnam, 2002). Con este estudio intentamos suplir las deficiencias anteriormente descritas, mediante una estrategia de muestreo que 1) considera proporcionalmente todos los servicios, 2) corrige los posibles sesgos por efecto del día, 3) establece el nivel de representatividad a partir de una Noche S y 4) corrige los resultados a través de un doble sistema de pesos. Debido a la amplitud de los datos recogidos, en el presente informe se incluyen exclusivamente los resultados más relevantes relacionados con las características sociodemográficas de los PSH, teniendo en cuenta por primera vez todos los controles muestrales mencionados.

\section{MÉTODO}

El presente trabajo se desarrolló en el área urbana de Madrid, que cuenta con una población de alrededor de tres millones de habitantes. En el momento de realizarse el estudio (1997) se disponía en Madrid de una red de recursos residenciales para PSH que incluía 10 albergues (públicos y privados) y se encontraban habilitados 2 centros especiales para albergar a PSH (Pabellón de Mayorales y estación de metro de Atocha) como dispositivos de emergencia durante períodos invernales, todos ellos al $100 \%$ de su capacidad. El total de plazas en los albergues y centros de acogida sumaban un total de 1086 plazas.

\section{Elaboración del listado de centros ${ }^{1}$ de atención a PSH en Madrid}

En primer lugar, se elaboró un listado que recogía los recursos y centros de atención a PSH existentes en Madrid mediante el siguiente procedimiento: 
1. Contacto con profesionales y responsables de organismos públicos y privados de atención a PSH. Se les solicitó que remitiesen tanto un listado de recursos y centros dependientes de su organismo como de cualquier otro conocido. A tal fin se contactó con: Cruz Roja Española, Comunidad Autónoma de Madrid, Excmo. Ayto. de Madrid, Grupo 5, C.E.D.I.A. (Centro de Acogida, Recepción e Información de Cáritas) y distintas ONGs.

2. Consulta de listados de recursos y centros existentes para personas seriamente excluidas aunque no fuesen específicos para PSH (ej. comedores sociales).

3. Recopilación de artículos en periódicos y revistas que informaban de recursos y centros existentes o de la apertura de nuevos, en especial publicaciones vinculadas con situaciones marginales (La Farola, La Calle...).

4. Contactos con parroquias. Con el objetivo inicial de ser exhaustivos, a través de la Guía Telefónica de Madrid 1996/97, se seleccionaron aleatoriamente una de cada cuatro parroquias, con las que se contactó telefónicamente para identificar si ofrecían servicios a PSH. Debe señalarse que las propias diócesis responsables no conocen los servicios ofrecidos por cada parroquia, servicios como dar bocadillos, servir de ropero, etc., de modo que no existían datos disponibles sobre este particular.

Con todas esta información, se elaboró un primer listado provisional que fue remitido a diferentes expertos, responsables políticos y profesionales de atención directa a PSH - con el objetivo de que revisasen la información recogida y enviasen sugerencias - tras lo que se elaboró el listado definitivo que incluía los siguientes apartados:

- Tipo de recurso (alojamiento, comedor, ropero, centro de información y acogida, baño público y otros).

- Teléfono, dirección y persona de contacto.

- Características.

- Actividades.

- $\mathrm{N}^{\circ}$ de plazas.

- Porcentaje de varones/mujeres.

- Criterios de inclusión/exclusión.

- $\mathrm{N}^{\circ}$ servicios por día.

\footnotetext{
' Se diferencia entre los términos recursos (conjunto de centros que ofrecen los mismos servicios, v.g.: comedores, albergues, etc.), centros (lugar especifico donde se ofrecen servicios a las PSH, v.g.: Albergue San Isidro, Comedor Maria Inmaculada, etc.) y servicios (cada una de las acciones ofrecidas en un centro, v.g.: $\mathrm{n}^{\circ}$ de camas, $\mathrm{n}^{\circ}$ de comidas servidas, etc.).
} 


\section{Elaboración de los instrumentos}

Se diseñaron dos instrumentos distintos: uno más sencillo, a modo de detección (screening), para la Noche S (Cuestionario 1), y otro más completo, a modo de entrevista estructurada, que incluía todos aquellos aspectos relevantes a la investigación (Cuestionario 2).

\section{Cuestionario 1 (ver anexo):}

En el diseño del primer cuestionario se siguieron los apartados utilizados habitualmente en muestreos de Noche S. Antes de abordar a los sujetos se reflejaba la hora exacta del contacto, el género, edad y procedencia estimada del entrevistado. A continuación se les invitaba a contestar sólo tres preguntas referentes a la utilización de recursos: la primera para identificar el uso de albergues durante las últimas 4 semanas; la segunda para identificar el uso de comedores en el mismo período, y una tercera, abierta, para identificar el uso de cualquier otro recurso durante dicho período de cuatro semanas.

\section{Cuestionario 2.}

Para el estudio final se elaboró un cuestionario que incluía una hoja de contacto y un abanico amplio de ámbitos de estudio. La selección de las variables e ítems se llevó a cabo según el siguiente procedimiento:

1. Revisión bibliográfica y documentación sobre estudios realizados en el área específica de PSH.

2. Contactos con algunos de los principales grupos de trabajo nacionales e internacionales. De esta forma se tuvo acceso a:

a) Cuestionarios utilizados en trabajos de investigación con PSH:

- Course of Homelessness Study Questionnaire (RAND Corporation)

- Course of Homelessness Study Follow-up Spanish Questionnaire (RAND Corporation)

- Metropolitan Area Drug Study. Washington, D.C. (Dennis, 1993).

- National Survey of Health and Stress (National Institute of Mental Health, USA)

- Hostels and Psl. n1364. (Meltzer, Londres).

- Enquête n ${ }^{\circ} 142$ (Institut National D’Etudes Demographiques, París).

- Cuestionario SMES (Salud Mental y Exclusión Social, Bruselas).

b) Estudios de ámbito nacional con población general:

- Estudios sobre problemas sociales y de actualidad en España (Fundación FOESSA).

- Estudio de las necesidades sociales en Aragón (Universidad Pública de Navarra). 
3. Elección de las variables objeto de estudio e ítems a evaluar en cada área (características demográficas, empleo y recursos económicos, salud física y mental, historia, sucesos vitales, apoyo social, utilización de servicios, derechos ciudadanos y expectativas de futuro) a partir de las diversas fuentes consultadas.

El tiempo necesario para la administración del cuestionario ${ }^{2}$ se estimó en aproximadamente 1 hora, realizándose a modo de entrevista estructurada.

\section{Utilización de una noche de muestreo: "NOCHE-S"}

El principal objetivo que se pretendía conseguir con este acercamiento era el de poder estimar el número de PSH que no utilizaban ningún tipo de recurso social (albergues, comedores, baños, roperos, etc.) durante un período determinado, en nuestro caso 4 semanas. La importancia de este dato es obvia si se pretende realizar un muestreo representativo de las PSH en una ciudad. El conocimiento de este dato permite estimar el porcentaje de PSH que se pierden al realizar distintos tipos de muestreos en recursos, igualmente permite realizar estimaciones sobre el número de PSH presentes en un período dado en un ámbito geográfico determinado.

La estrategia de Noche $\mathrm{S}$ ha sido utilizada para identificar el mayor número posible de PSH en áreas urbanas que en una noche dada se encuentran durmiendo literalmente en la calle. La estrategia básica consiste en realizar un recorrido por las calles de la ciudad identificando a todas aquellas personas que se encuentran durmiendo en la misma. Para ello se escoge una noche de máxima ocupación en los recursos de la ciudad (generalmente noches de frío intenso) y se recorren un número determinado de calles o áreas de la misma. La selección de áreas puede hacerse mediante procedimientos aleatorios por distritos o manzanas (más útiles en zonas urbanas con un gran número de $\mathrm{PSH}$ ) o mediante procedimientos guiados en los que se seleccionan aquellas calles o áreas que reúnen una mayor concentración de PSH (más útiles en zonas urbanas con números pequeños de PSH). Los cuestionarios utilizados deben ser muy sencillos. Dada la situación y la hora en que se realiza el contacto, generalmente se reducen a una sencilla Hoja de Contacto con algunas preguntas muy breves y/o observaciones del entrevistador.

En este caso nuestra opinión fue coincidente con la de los expertos externos consultados en el sentido de utilizar una estrategia de muestreo guiada. Se consideró oportuno que la actividad tuviese lugar en período invernal y evitando que coincidiese con un fin de semana o día festivo porque en estos días se dificulta

\footnotetext{
${ }^{2}$ Este cuestionario puede ser solicitado a los autores.
} 
RIS

REVISTA INTERNACIONAL DE SOCIOLOGIA

No 32, Mayo-Agosto, 2002

M. MUÑOZ, C. VÁZQUEZ, JJ. VÁQUEZ, A.B. GUISADO Y M. CRESPO

su localización. Finalmente se seleccionó la noche del martes 20 al miércoles 21 de enero de 1997 y se utilizó el Cuestionario 1 ya descrito.

Selección de las áreas de muestreo y distribución de los equipos de entrevistadores

De acuerdo a la estrategia guiada, las áreas de muestreo se seleccionaron (con la intención de localizar la mayor cantidad de PSH posible) a partir de la información procedente de:

- Reuniones con expertos.

- Experiencia del equipo en investigaciones anteriores con PSH (Muñoz, Vázquez y Cruzado, 1995).

- Realización de una "Noche piloto" previa - miembros del equipo de investigación, en compañía de personal de la UMES (Unidad Móvil de Emergencia Social del Ayuntamiento de Madrid gestionada por el Grupo 5), recorrieron zonas susceptibles de ser utilizadas para pernoctar por PSH.

En función de toda esta información se establecieron tres áreas de muestreo para la "noche - S" que trataban de incluir todas aquellas zonas en las que la concentración nocturna de PSH era más alta. Paralelamente se organizaron tres equipos de entrevistadores que se distribuyeron las distintas áreas de la siguiente forma:

Equipo 1: recorrió las zonas Alrededores de la antigua Estación Sur de Autobuses, Plaza de Colon y aledaños y Calle Alenza y alrededores.

Equipo 2: recorrió las zonas Calle de la Princesa, Plaza del Callao, Calle del Carmen, Calle Preciados, Puerta del Sol y Calle Atocha, hasta la Glorieta de Carlos V.

Equipo 3: recorrió las zonas Zona de Azca, Calle del Dr. Esquerdo y aledaños y Calle de Sainz de Baranda y alrededores.

Estrategias de abordaje y criterios de exclusión de la muestra

Cada equipo de entrevistadores realizó un "barrido" (entre las 22:00 y las 3:30 horas) por las zonas indicadas buscando a todas aquellas personas que manifestaban actitudes indicativas de disponerse a pasar la noche en la calle (agrupando cartones, cubriéndose de ropa, acondicionando lugares, disponiéndose a cenar en la calle...), o que presentaban evidentes muestras de pernoctar en ésta. Una vez localizados los potenciales sujetos, un miembro del equipo se dirigía a ellos $y$, tras ofrecerles un café caliente, les solicitaba contestar al breve cuestionario. Del total de personas abordadas el $60,3 \%$ (38) aceptaron contestar a la entrevista, el $4,8 \%$ (3) rehusaron hacerlo y el $33,3 \%$ (21) fueron considerados no aptos para ello. 
Se consideraron "no aptos" los sujetos profundamente dormidos que no atendieron a los requerimientos de los entrevistadores, los sujetos que presentaban un grave deterioro cognitivo o aquellos con elevados niveles de confusión aparentemente derivados de una intoxicación por alcohol $u$ otras sustancias psicoactivas.

\section{Resultados}

En la tabla 1 se reflejan los datos referentes a las respuestas al Cuestionario 1 en relación con el uso de recursos para PSH en las últimas 4 semanas.

Si se asume que la suma del total de plazas ocupadas en albergues en aquel momento (todas las disponibles estaban ocupadas $=1086$ ) y las personas entrevistadas en la calle durante la Noche $S(n=62)$ representan un muestreo representativo del universo de PSH que aquella noche había en Madrid, entonces se dispone de una muestra total de 1.149 PSH. De éstos:

a) 1.098 PSH (el 95,5\%) utilizaron albergues en las últimas 4 semanas.

b) 1.115 PSH (el 97,0\%) utilizaron albergues o comedores sociales en las últimas 4 semanas.

c) 1.119 PSH (el 97,4\%) utilizaron albergues, comedores sociales o baños en las últimas 4 semanas.

d) 1.120 PSH (el 97,5\%) utilizaron albergues, comedores sociales, baños o roperos en las últimas 4 semanas.

Tabla 1.

Utilización de servicios en las últimas cuatro semanas por parte de las personas sin hogar contactadas en la Noche-S.

\begin{tabular}{lc}
\hline Servicios & $\begin{array}{c}\text { Porcentaje de usuarios } \\
\mathrm{N}=62\end{array}$ \\
& \\
Albergues & $31,6 \%$ \\
Comedores & $63,2 \%$ \\
Baños & $23,7 \%$ \\
Roperos & $13,2 \%$ \\
Servicios sanitarios & $2,6 \%$ \\
Servicios de salud mental & - \\
UMES & - \\
CEDIA & - \\
Parroquias & $5,3 \%$ \\
Otros alojamientos & - \\
Otros & $5,3 \%$ \\
Ns/nc, no apto & $38,1 \%$ \\
\hline
\end{tabular}


RIS

REVISTA INTERNACIONAL DE SOCIOLOCÍA

N॰ 32, Mayo-Agosto, 2002

M. MUÑOZ, C. VÁZQUEZ, J.J. VÁOUEZ, A.B. GUISADO Y M. CRESPO

De este modo puede concluirse que realizando un muestreo que incluyese todos los días de la semana, la estratificación por tipo de recurso en albergues y comedores y que se prolongase durante 4 semanas o tuviera en cuenta este período en la redacción de las preguntas del cuestionario, se accedería al $97 \%$ de las PSH en el caso de Madrid. Ampliar el muestreo a los estratos de baños sociales y roperos solamente aumentaría un $0,5 \%$ de representatividad, aumentando muy significativamente los gastos y la complejidad del análisis matemático posterior. En función de lo anterior nuestro equipo decidió llevar a cabo el muestreo para el estudio final en albergues y comedores exclusivamente, todos los días de la semana y durante un periodo de cuatro semanas.

\section{ESTRATEGIAS DE MUESTREO: MUESTREO EN ALBERGUES Y COMEDORES}

\section{Características del muestreo}

En función de todo lo anterior se decidió realizar una encuesta con las siguientes características:

a) Población (universo) de estudio. Todas aquellas personas que cumplan la definición de PSH de FEANTSA en el momento de la entrevista, mayores de 18 años y que utilicen los recursos para PSH durante ese mismo período. De este modo se obtiene una muestra representativa del $97 \%$ de todas las PSH que haya en Madrid durante el período de la encuesta.

b) Marcos muestrales. Se eligieron los dos marcos muestrales que mantenían una mejor relación coste/beneficio, es decir: albergues para PSH y comedores sociales. Se utilizó en ambos casos el listado exhaustivo de recursos confeccionado anteriormente.

c) Tamaño de la muestra. Dado que el número total de PSH es desconocido, el tamaño de la muestra se calculó sobre el universo de servicios por día que ofrecían los distintos recursos. En el caso de los albergues las plazas totales en aquel momento eran 1086. En el caso de los comedores el número de comidas servidas diariamente era de 1080 , considerando que existe un número desconocido de duplicidades entre ambas cantidades. En función de lo anterior se utilizó la fórmula genérica para obtención de una muestra aleatoria para poblaciones finitas. El tamaño resultante para un nivel de confianza del $95 \%$ fue de $\mathrm{N}=300$.

d) Aspectos temporales. Se eligió un marco temporal restrictivo de cuatro semanas entre febrero y marzo de 1997 . Se realizaron encuestas todos los días y para corregir el posible sesgo entre días festivos y laborables se estableció una distribución proporcional del día de la encuesta en cada centro: $70 \%$ de contactos en días laborables y $30 \%$ en sábados, domingos y días festivos. Esta proporción responde a los porcentajes de servicios ofrecidos en conjunto en ambos tipos de días. 
e) Aleatorización y proporcionalidad. La muestra se eligió (ver punto siguiente, relativo a la recogida de datos) de acuerdo a los principios de aleatoriedad y proporcionalidad. Es decir, la muestra se distribuyó proporcionalmente al número de servicios/día de cada centro de forma aleatoria y, posteriormente, la persona usuaria de cada servicio (cama, comida) era seleccionada en el centro de modo, igualmente, aleatorio.

De este modo se seleccionó una muestra con un tamaño de $\mathrm{N}=300$. Posteriormente se realizó una selección a partir del listado completo de albergues y comedores que incluía el número de servicios que ofrecía cada centro. De este modo se pudo llevar a cabo una asignación de unidades muestrales $(\mathrm{N}=3)$ a cada centro en función de su tamaño mediante un procedimiento aleatorio, y posteriormente calcular el número total de entrevistas a realizar en cada centro y en cada tipo de recurso. Los resultados de dicha asignación indicaron que el $54 \%$ de las entrevistas debían ser realizadas en albergues y el $46 \%$ en comedores $(n=162$ y $\mathrm{n}=138$ respectivamente). El período de muestreo sería de 4 semanas.

\section{Recogida de información y análisis de datos}

a) Entrevistadores. Como entrevistadores se utilizó un equipo de 15 estudiantes de últimos cursos de Psicología que recibieron un entrenamiento específico en la utilización del cuestionario y en el trato social con PSH y personas que padecen procesos de marginación en general. Las entrevistas eran revisadas por un equipo de 4 supervisores para asegurar la correcta cumplimentación de cada cuestionario y optimizar la fiabilidad y validez de los datos.

b) Muestreo de los recursos. En primer lugar se decidió utilizar 100 unidades de muestreo de 3 elementos cada una (cada unidad de muestreo responde al número de entrevistas que consideramos posible realizar en un centro en un día dado). Sobre la base del listado de recursos elaborado anteriormente se realizó una distribución proporcional y aleatoria (lista de números aleatorios) de las 100 unidades de muestreo. En el caso de los albergues se realizó en función del número de camas de cada centro; en el caso de los comedores en función del número de comidas servidas diariamente en cada centro.

c) Selección de los entrevistados. Una vez conocido el número de entrevistas a realizar en cada centro, la selección de las personas se realizó, igualmente, de forma aleatoria. Obviamente el procedimiento exacto fue distinto en cada centro debido a sus características concretas. En aquellos en los que se disponía de listados se utilizaron tablas de números aleatorios, en los que no se disponía de listados se prefirió utilizar una razón sobre el orden de entrada al centro a partir de la llegada de los entrevistadores. Una vez seleccionado, el entrevistador iniciaba el contacto, confirmaba los criterios de inclusión en la muestra, cumplimentaba la 
RIS

REVISTA INTERNACIONAL DE SOCIOLOGÍA

No 32, Mayo-Agosto, 2002

M. MUÑOZ, C. VÁZQUEZ, JJ. VÁQUEZ, A.B. GUISADO y M. CRESPO

Hoja de Contacto del Cuestionario 2 y solicitaba la aceptación del entrevistado. Al finalizar la entrevista los entrevistados recibían un "Metro-Bus" por su colaboración, de lo que no se advertía al entrevistado hasta finalizar la entrevista. Todas las entrevistas fueron anónimas. El porcentaje de negativas a efectuar la encuesta estuvo en el 30\%, lo que se asemeja a lo obtenido en otras ciudades europeas con estudios similares (Marpsat y Firdieu, 1998).

d) Muestra teórica vs. muestra empírica. Como se ha indicado, la muestra teórica estaba compuesta por 300 cuestionarios, de los que el $54 \%$ (162) se debían realizar en albergues y el $46 \%$ (138) en los comedores. Finalmente se realizaron 289 entrevistas, el 54,3\% (157) en albergues y el $45,7 \%$ (132) en comedores.

La diferencia entre los tamaños teóricos y empíricos se corrigió con un coeficiente de ajuste aplicado para cada centro:

$$
\text { Coeficiente de Ajuste }=\frac{\text { Número empirico de cuestionarios en el centro }}{\text { Número teórico de cuestionarios en el centro }}
$$

De este modo se contemplan coeficientes de ajuste específicos para cada uno de los centros incluidos en el muestreo, coeficientes que se van a incluir posteriormente en las fórmulas aplicadas para la ponderación de las variables.

e) Ponderación en función de la probabilidad de elección de un servicio en cada centro. El primer factor de ponderación se establece en función de la probabilidad que tiene una persona de ser incluido en la muestra en cada centro. Esta primera ponderación garantiza la representatividad de la muestra independientemente del tamaño del centro y recurso en que ha sido seleccionada. Siguiendo el esquema de muestreo esta probabilidad está en función directa del tamaño del centro.

$\mathbf{P}_{\text {servicio y centro }}=\quad$ Probabilidad de selección del servicio en el centro

Esta probabilidad es distinta para cada centro y se expresa:

$\mathbf{P}_{\text {Servicio y centro }}=\frac{N^{o} \text { unidades muestrales del centro } x \text { Tamaño de la unidad muestral }}{\text { Número de servicios del centro }}$

simplificando,

$\mathbf{P}_{\text {Servicio y centro }}=\frac{N^{\circ} \text { teórico de entrevistas en el centro }}{\text { Número de servicios en el centro }}$ 
La ponderación se establece como la función inversa de la probabilidad de ser incluido en la muestra, corregida por la desviación de la muestra empírica respecto a la teórica en cada centro, de este modo:

$$
\text { Peso }_{\text {servicio }}=\quad \frac{1}{P_{\text {servicio }} \times \text { Coeficiente de ajuste }}
$$

f) Ponderación en función de la probabilidad de elección de un persona en función de su utilización de recursos. Hasta el momento, tanto el muestreo como el factor de corrección (ponderación) se ha centrado en los servicios ofrecidos en cada centro, por tipo de recurso muestreado (albergues y comedores). Sin embargo la probabilidad de ser elegida de una persona está en función de la probabilidad de elección del servicio que está recibiendo (en el que es contactada dicha persona) ý de la probabilidad de la persona de ser contactada por duplicado en otro servicio. Esta segunda probabilidad está en función directa de la utilización de otros centros por parte de la propia persona. En nuestro caso al realizar la entrevista en un tipo de recurso (albergue o comedor) la persona solamente puede ser elegida en el otro tipo de recurso. Es decir, si una persona es elegida en un albergue un día dado, la probabilidad de volver a ser elegida ese mismo día queda reducida a la probabilidad de volver a ser elegida en un comedor. Esta última probabilidad estará en función directa de la utilización o no de comedores por esa persona ese día. Para conocer estas probabilidades se incluyeron preguntas en el cuestionario relativas a la utilización de recursos en las 24 horas inmediatamente anteriores a la entrevista. De este modo, el estudio permite realizar estimaciones sobre las personas sin hogar un día promedio del periodo de muestreo al permitir estimar el número de personas que utilizan los dos tipos de recurso el mismo día. A continuación se exponen los algoritmos aplicados a aquellas personas que habían utilizado ambos tipos de recursos durante las últimas 24 horas.

$$
\begin{aligned}
& \begin{array}{l}
\text { Pesopersona entrevistada en } \\
\text { albergue }
\end{array} \\
& \begin{array}{l}
\text { Pesopersona entrevistada } \\
\text { en comedor }
\end{array} \\
& \text { donde, }
\end{aligned}
$$

$$
\mathbf{P}_{\text {albergues }}=\frac{N^{o} \text { de entrevistas realizadas en albergues }}{N^{o} \text { total de servicios en albergues }}
$$


RIS

REVISTA INTERNACIONAL DE SOCIOLOGIA

No 32, Mayo-Agosto, 2002

M. MUÑOZ, C. VÁZQUEZ, J.J. VÁQUEZ, A.B. GUISADO y M. CRESPO

$\mathbf{P}_{\text {comedores }}=\frac{\mathrm{N}^{\circ} \text { de entrevistas realizadas en comedores }}{\mathrm{N}^{\circ} \text { total de servicios en comedores }}$

g) Análisis de datos. Los análisis de datos que se incluyen a continuación se centran en la estimación de porcentajes ponderados sobre el total de la muestra de acuerdo al procedimiento descrito anteriormente. Para el proceso de los datos se ha utilizado el programa SPSS. Los análisis de las diferencias entre porcentajes de las variables nominales se ha realizado utilizando el estadístico "Chi Cuadrado". Para comparar los datos ponderados con los porcentajes y medias hallados al analizar los datos sin aplicar la fórmula de ponderación, se han tomado como valores teóricos éstos últimos. En el caso de las variables continuas se han aplicado "Pruebas t-Student" para medidas dependientes.

\section{RESULTADOS}

A continuación, y con el objetivo de ilustrar el procedimiento descrito, se recogen los principales resultados encontrados en el presente estudio con relación a las variables sociodemográficas más relevantes para una primera aproximación a la caracterización de las PSH en Madrid. En primer lugar, se presenta una comparación de los valores de las principales variables en la muestra final con respecto a los encontrados en la Noche S. Posteriormente se resumen los resultados agrupados en variables descriptivas y variables de situación laboral 0 actividad económica.

\section{Comparación entre las PSH entrevistadas en la "Noche-S" y las PSH entre- vistadas en los centros}

Se han analizado las diferencias existentes entre las dos muestras obtenidas en la Noche $\mathrm{S}$ y en el muestreo de servicios. Este análisis se realiza sobre las variables más relevantes y tiene como principal objetivo el establecimiento de la correspondencia entre ambas muestras. Como puede observarse en la tabla 2, ambas muestras resultan equivalentes en las variables estudiadas.

\section{Descriptivos de las PSH de Madrid}

A continuación se llevó a cabo un análisis descriptivo de las variables sociodemográficas más importantes. En la tabla 3 se presentan los datos relativos al tiempo medio que llevan las personas de la muestra en la situación de Sin Hogar, el género, la edad media, el lugar de nacimiento (origen), el estado civil, el número de hijos nacidos vivos, las personas con las que convive en la actualidad (aún en su situación Sin Hogar) y el máximo nivel escolar alcanzado. 
Tabla 2.

Género, edad y procedencia de las PSH contactadas y entrevistadas durante la noche $-S$, en albergues y comedores sociales.

\begin{tabular}{lcc}
\hline & $\begin{array}{c}\text { Comedores sociales y } \\
\text { albergues } \\
\mathrm{n}=289\end{array}$ & $\begin{array}{c}\text { Noche S } \\
\mathrm{n}=38\end{array}$ \\
\hline Género & & \\
$\quad$ Varones & $86,9 \%$ & $82,1 \%$ \\
$\quad$ Mujeres & $13,1 \%$ & $17,9 \%$ \\
Edad & $4,5 \%$ & - \\
$\quad$ Menores de 20 años & $53,6 \%$ & $59,0 \%$ \\
Entre 21 y 39 años & $35,3 \%$ & $35,9 \%$ \\
Ente 40 y 59 años & $6,6 \%$ & $5,1 \%$ \\
Mayores de 60 años & & $84,6 \%$ \\
Procedencia & $77,6 \%$ & $7,7 \%$ \\
Españoles & $22,4 \%$ & \\
Extranjeros & & \\
\hline
\end{tabular}

${ }^{*} \mathrm{p}<0,05$

\section{Situación laboral}

En relación a la vida laboral de las PSH se han analizado los datos relativos a la vida laboral media - expresada en el número de meses que han mantenido un empleo, sumando todos los periodos a lo largo de toda su vida-, la situación laboral actual y el tiempo que lleva en situación de desempleo. La actividad económica realizada "sin empleo" incluye diversas actividades que reportan un beneficio económico a la persona, pero que no están reglamentadas, ni se consideran empleos, como por ejemplo vender pañuelos en un semáforo, ejercer la prostitución, mendigar en una iglesia, etc. En la tabla 4 se incluyen los porcentajes correspondientes a estas variables.

\section{CONCLUSIONES}

Los datos presentados en este informe permiten abordar dos tipos de conclusiones: por un lado, aquellas referentes a las repercusiones metodológicas del trabajo y, por otro, las relativas a los datos identificados en las PSH en Madrid. En lo relativo a los aspectos metodológicos debe señalarse en primer lugar la equivalencia 
RIS

REVISTA INTERNACIONAL DE SOCIOLOGI

Tabla 3.

Principales descriptivos de las PSH en Madrid

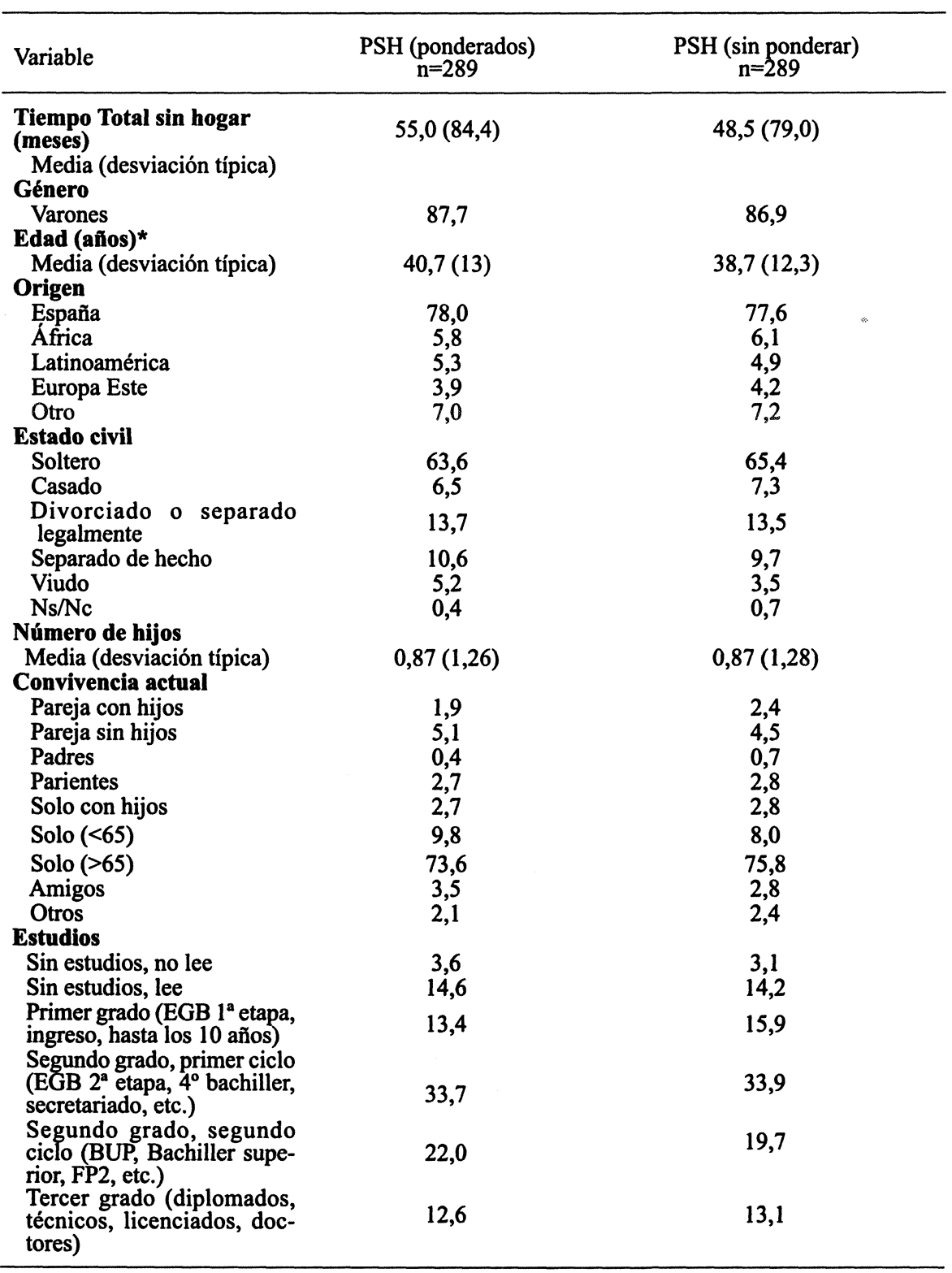

${ }^{*} \mathrm{p}<0,05$

170 
Tabla 4.

Datos relativos a la vida laboral de los PSH en Madrid.

\begin{tabular}{lcc}
\hline Actividad laboral & $\begin{array}{c}\text { PSH } \\
\text { (ponderados) } \\
\mathrm{n}=289\end{array}$ & $\begin{array}{c}\text { PSH } \\
\text { (sin ponderar) } \\
\mathrm{n}=289\end{array}$ \\
\hline Duración total vida laboral (meses) & 122,6 & 109,6 \\
$\quad$ Media (desviación típica) & $(141,8)$ & $(130,1)$ \\
Empleo (contratados o autónomos) & & \\
Con empleo & $2,6 \%$ & $2,5 \%$ \\
Sin empleo 0-3 meses & $6,9 \%$ & $8,8 \%$ \\
Sin empleo 4-6 meses & $7,2 \%$ & $8,4 \%$ \\
Sin empleo 7-11 meses & $3,0 \%$ & $3,4 \%$ \\
Sin empleo 1-2 años & $13,1 \%$ & $13,4 \%$ \\
Sin empleo 2 o más años & $66,7 \%$ & $62,6 \%$ \\
Ns/Nc & $0,5 \%$ & $0,8 \%$ \\
Actividad económica última semana (sin contrato & & \\
ni en régimen de autónomos) & $18,6 \%$ & $16,6 \%$ \\
Prostitución & $0,7 \%$ & $0,7 \%$ \\
Mendicidad & $3,0 \%$ & $2,4 \%$ \\
Pensiones, prestaciones y ayudas oficiales & $25,3 \%$ & $23,3 \%$ \\
Desempleo & $3,3 \%$ & $3,8 \%$ \\
IMI & $10,2 \%$ & $9,0 \%$ \\
\hline
\end{tabular}

${ }^{*} \mathrm{p}<0,05$.

encontrada entre las muestras de PSH entrevistados en la calle durante la Noche-S y los entrevistados en albergues y comedores. El hecho de que ambas muestras resulten equiparables en variables como género, edad y procedencia, hace pensar que las personas que un día cualquiera están durmiendo en la calle en Madrid pertenecen a la misma población que las que pueden encontrarse ese mismo día en los albergues o comedores de la capital. Este dato, unido al hecho de que el muestreo en albergues y comedores engloba al $\mathbf{9 7 \%}$ de las PSH si se continúa durante un periodo de 4 semanas, justifica plenamente la alternativa de muestreo realizada en este trabajo. Es decir, parece que un muestreo aleatorio llevado a cabo en albergues y comedores y continuado durante un periodo suficiente, es la mejor alternativa, en términos de coste/beneficio, para identificar las características que afectan a este grupo de población, al menos en la ciudad de Madrid (que ofrece una red relativamente amplia de recursos). Creemos que este hallazgo es relevante puesto que permite utilizar estrategias de muestreo sencillas que aseguran, al menos mientras no cambien significativamente las condiciones sociodemográficas de la población, la representatividad de los estudios siempre que se sigan las pautas 
RIS

REVISTA INTERNACIONAL DE SOCIOLOGIÁ

№ 32, Mayo-Agosto, 2002

M. MUÑOZ, C. VÁZQUEZ, JJ. VÁQUEZ, A.B. GUISADO y M. CRESPO

de muestreo que hemos indicado (foco temporal del muestreo y/o de las preguntas en 4 semanas o más, y muestreo de albergues representativo).

Un segundo aspecto metodológico hace relación a la necesidad de incluir un sistema de ponderación para los datos obtenidos siguiendo la estrategia de muestreo indicada anteriormente. Si se observan las tablas 3 y 4 y se analizan las diferencias entre las columnas ponderadas y sin ponderación puede pensarse que los datos son tan similares que el esfuerzo realizado para llevar a cabo la ponderación no sería necesario. Nada más alejado de la realidad. En primer lugar, aparecen diferencias significativas entre ambos tipos de datos en variables clave como la edad. En segundo lugar, se identifican tendencias que, sin llegar al nivel de significación estadística, pueden influir decisivamente en análisis más elaborados (i.e., análisis multivariados). En tercer lugar, en nuestro caso debe esperarse una mayor proximidad de los datos ponderados y sin ponderación debido al escaso margen de desviación conseguido en el muestreo empírico con respecto al muestreo teórico planeado. En este mismo sentido, la lista completa de recursos y centros, y la verosimilitud de los datos referidos a cada uno de ellos, hace que la estrategia de muestreo presente, en sí misma, una representatividad de la población muy importante. En muestras con mayores desviaciones y menor nivel de representatividad de centros (bien por el tamaño, la localización geográfica, u otros factores) los sistemas de ponderación resultarían aún más importantes.

Aunque no es el objetivo principal del presente trabajo, los datos elaborados hasta el momento permiten adelantar una panorámica general sobre alguna de las principales características que afectan a esta población.

En lo relativo a los descriptores sociodemográficos incluidos en la tabla 3, el primer dato que merece atención es el de la alta cronicidad de la situación (la media se sitúa por encima de los 10 años en la situación de sin hogar), lo que contrasta con lo observado en países como Estado Unidos, en que la cronicidad es menor y se observa en mayor medida el fenómeno de puerta giratoria (Muñoz, Vázquez, Koegel et al., 1998). Este dato parece indicar, igualmente, el fracaso de las medidas de reinserción social, al menos, con un fragmento de la población sin hogar. Igualmente, este patrón de edad puede obedecer al hecho de que el sistema de protección familiar y de redes naturales de apoyo en nuestro país tiene un gran peso en la amortiguación de las dificultades económicas y sociales (Vázquez, Muñoz y Rodríguez, 1999). De este modo, parece que en nuestro país sólo personas en circunstancias psicosociales muy extremas pueden llegar a la condición de PSH y desde ese punto de arranque, las condiciones de reinserción se presentan más complicadas. De hecho, algunos estudios previos han indicado que existe un número abrumadoramente elevado de estresores y dificultades sociales de las PSH antes del primer episodio en sus vidas de quedarse sin hogar (Muñoz, Vázquez, Bermejo y Vázquez, 1999). En definitiva, el perfil de las PSH en nuestro país parece tener unas características distintivas al menos en la comparación con el existente en países anglosajones. 
Un segundo aspecto que merece atención en este capítulo es el relativo a la edad y género de las PSH. La opinión mayoritaria entre los profesionales de atención directa a esta población indican que se identifica una tendencia a la feminización y a la mayor juventud de la población sin hogar. Nuestros datos, a pesar de no poder contar con comparaciones basadas en series temporales anteriores representativas, no parecen identificar esta tendencia. Los porcentajes de varones siguen estando por encima del $85 \%$ y la edad media se mantiene en torno a los 40 años, lo que coincide con estudios anteriores en nuestra ciudad (Cáritas, 1975; Muñoz et al., 1995). Sin embargo sí aparece una sobrerepresentación de personas inmigrantes en la población sin hogar, si se compara con la población general en nuestro país. La cifra de PSH que proceden de otros países se sitúa por encima del $22 \%$, lo cual es especialmente relevante si se considera la peligrosidad que presenta esta situación para quienes carecen de documentación en regla (su identificación generalmente comporta la deportación) y las redes de apoyo que pudieran existir entre este tipo de poblaciones. De nuevo el fracaso de los recursos planificados para la integración social de los inmigrantes en nuestra ciudad aparece como un obstáculo que se puede poner de manifiesto en esta tendencia creciente. Sin duda se necesitarán recursos o políticas específicas de atención a estas personas puesto que sus condiciones sociales, económicas y psicológicas no parecen equivalentes a las del grupo medio de PSH de nuestro país.

Los factores de aislamiento social que repetidamente se nombran al referirse a las PSH aparecen perfectamente reflejados en la variable de número de hijos (por debajo de la media de la población general) y, muy especialmente, en la variable de convivencia actual. Una enorme mayoría, por encima del $83 \%$, viven solos. Nuestra opinión es que este tipo de información merece un abordaje más amplio, considerando un mayor número de variables descriptivas de las redes sociales de estas personas, incluyendo aquellas relacionadas con su proceso de socialización, que permitan avanzar más firmemente en el estudio de la importancia de las relaciones sociales en la génesis y mantenimiento de este tipo de situaciones de máxima exclusión social, como se ha hecho en el estudio de otros procesos psicológicos (Weissman y Markowitz, 1992 ; Herman et al., 1997; Brown, 1998). De hecho, este aislamiento parece una de las claves del mantenimiento de la situación de PSH y los programas de rehabilitación más eficaces siempre inciden en el fortalecimiento de estas redes de apoyo y comunicación como un elemento imprescindible rehabilitador.

La agrupación de factores educativos (tabla 3 ) y laborales (tabla 4) permite acceder a una panorámica interesante, aunque necesariamente reducida, sobre la formación y actividad económica de las PSH. Puede observarse un nivel de formación bajo en estas personas: casi uno de cada tres no ha terminado la educación obligatoria, aunque pueda sorprender el porcentaje de universitarios, cercano al de la población general. Este dato, aunque apunta a la falta de formación como un posible factor de incidencia en la génesis de este problema, no puede ser extra- 
polado fácilmente y merece un estudio más detenido en trabajos posteriores. En este mismo sentido deben interpretarse los datos incluidos en la tabla 4, referentes a la actividad económica de las PSH. La inmensa mayoría ha realizado actividades laborales durante varios años: la media se sitúa en 10 años de vida laboral previa a su situación SH. Parece que la situación de desempleo, que afecta a la práctica totalidad, tiene un papel determinante en la etiología del problema SH. Encontramos que 2 de cada 3 PSH sufren una situación de desempleo de larga duración (más de dos años). Sin embargo, casi un 20\% de las PSH entrevistadas realizan alguna actividad económica alternativa para sobrevivir. Por cierto, resulta relevante indicar que, pese al estereotipo social existente, la prostitución y la mendicidad juegan un papel relativamente escaso. Es decir, la identificación de PSH con mendigo o prostituta es completamente inadecuada: sólo el 3\% de las PSH ejercen la mendicidad y no llegan al $1 \%$ las PSH que ejercen la prostitución. Como se ha observado en estudios cualitativos anteriores, situaciones como la mendicidad generalmente son vividas con vergüenza o autorreproche y hay una resistencia casi siempre activa para ejecerla incluso en situaciones tan extremas de pobreza (Muñoz, Vázquez y Vázquez, 1999).

Siempre siguiendo sus autoinformes debe remarcarse que una de cada cuatro PSH recibe alguna prestación o pensión económica, incluyendo desempleo o salarios de integración social o no contributivos (ej.: IMI). Estos datos nos colocan ante un grupo de personas que han disfrutado de trabajo durante varios años y que, al perderlo, recurren a las prestaciones económicas (pensiones, etc.) y a actividades económicas no regladas para sobrevivir (venta de pañuelos, recogida de cartones, etc.), además de a los recursos para PSH (albergues y comedores principalmente). Sin embargo, estas estrategias se muestran claramente ineficaces para permitirles salir de esa situación, pues son muy pocos los que recuperan un trabajo y, posiblemente menos, los que recuperan un hogar.

No queremos finalizar sin señalar que la complejidad de la situación $\mathrm{SH}$ necesita de aproximaciones igualmente complejas a su realidad. Pensamos que trabajos como el presentado pueden ayudar a establecer la necesidad de realizar un estudio más detenido y completo de los agentes causales y mantenedores de este tipo de situación antes de iniciar la planificación de recursos y políticas destinadas a solventar el problema. Todavía se necesita una mejoría en la metodología de acercamiento a esta población que permita conocer mejor un fenómeno, por otra parte, tan visible. Es imprescindible obtener una información más directa sobre el uso y necesidades de servicios de las personas en la situación SH a la vez que se debe avanzar en el conocimiento teórico sobre los factores socioeconómicos y psicosociales que favorecen la aparición, el mantenimiento y, aun siendo un tema aún más desconocido, la desaparición de este tipo de situaciones. Finalmente, quisiéramos dejar constancia de la necesidad de que las ciencias sociales se aproximen al análisis de la exclusión social desde el rigor científico. Con mucha frecuencia, los estudios sobre estas áreas son anecdóticos, accidentales, 
o claramente sesgados. La planificación de políticas de intervención claramente debe anclarse en un mejor y más exacto conocimiento de la realidad y esperamos que análisis como el que se presenta en este estudio contribuya de algún modo a ese objetivo.

\section{REFERENCIAS}

AYUNTAMIENTO DE MADRID (1986), Transeúntes e Indigentes, Madrid, Concejalía de Servicios Sociales.

AVRAMOV, D. (1995), Homeless in the European Union Social and Legal Exclusion in the 1990s, Bruselas, European Federation of National Organizations Working with the Homeless (FEANTSA).

BROWN, G.W. (1998), "Genetic and Population Perspectives on Life Events and Depression", Social Psychiatry and Psychiatric Epiedmiology, $\mathrm{n}^{\circ} 33$ (8), pp. 363-372.

BURMAN, M.A. y P. KOEGEL (1988), "Methodology for Obtaining a Representative Sample of Homeless Persons", Evaluation Review, n 12, pp. 117-152.

BURT, M. (1992), Practical Methods for Counting Homeless People, Washington D.C., Interagency Council for Homeless and Department of Housing and Urban Development.

BURT, M.R. (1993), Exploring a Service-based Methodology: Types of Service to Include, Paper presented at the Towards Census 2000: Research Issues for Improving Coverage of the Homeless Population conference, Washignton, DC, U.S. Department of Commerce, Bureau of the Census, September.

CABRERA, P.J. (1998), Huéspedes del aire. Sociologia de las personas sin hogar en Madrid, Madrid, Universidad Pontificia de Comillas.

CÁRITAS ESPAÑOLA (1997), Politicas contra la exclusión social, Madrid, Cáritas Española.

COUSINEAU, M. y T. WARD (1992), "An Evaluation of the S-Night Street Enumeration of the Homeless in Los Angeles", Evaluation Review, n 16 (4), pp. 389-399.

DALY, M. (1994), The Right to a Home, the Right to a Future. Thrid Report of the European Observatory on Homelessness, 1994 (3), Bruselas, FEANTSA.

DENNIS, M.L. (1993), Coverage of a Service-based Methodology: Findings from the DC MADS Homelessness Study, Paper presented at the Towards Census 2000: Research Issues for Improving Coverage of the Homeless Population conference, Washignton, DC, U.S. Department of Commerce, Bureau of the Census, September.

DEVINE, J. y J. WRIGHT (1992), “Counting the homeless: S-Night in New Orleans”, Evaluation Review, $\mathrm{n}^{\circ} 16$ (4), pp. 409-417. 
RIS

REVISTA INTERNACIONAL DE SOCIOLOGI

N 32, Mayo-Agosto, 2002

M. MUÑOZ, C. VÁZQUEZ, JJ. VÁQUEZ, A.B. GUISADO y M. CRESPO

EDIN, K. (1992). "Counting Chicago's Homeless: An Assessment of the Census Bureau's "Street and Shelter Night", Evaluation Review, no 16 (4), pp. 365-375.

FIRDION, J.M. (1995), "Les Sans-abri et l'Enquetes Statistique", Fondations, n 1, pp. 21-46.

GRUPO 5 (1994), Informe 1994 de las UMES, Madrid, Ayuntamiento de Madrid.

HERMAN, D.B, E.S. SUSSER, E.L. STRUENING y B.L. LINK (1997), "Adverse Childhood Experiences: Are They Risk Factors For Adult Homelessness ?", American Journal of Public Health, $\mathrm{n}^{\circ} 87$ (2), pp. 249-255.

HOPPER, K. (1992), "Counting the Homeless: S-Night in New York", Evaluation Review, n 16 (4), pp. 376-388.

INFANTE, E.J., G.C. JERÓNIMO, E.S. MARTÍN, M.B. MAYORAL, J.R. MORILLA y R.A. SALVAREZ (1990), Marginados y excluidos en las calles de Madrid, Madrid, Consejeria de Integración Social.

KOEGEL, P. (1992), “Understanding Homeless: An Etnographic Approach”, en R.I. Jahiel (coord.), Homeless. A prevention-Oriented Approach, Baltimore, The Johns Hopkins University Press.

KOEGEL, P. y A. BURNAM (1992), "Problems in the Assesment of Mental Illness among the Homeless: An Empirical Approach", en M.J. Robertson y M. Greenblatt (coord.), Homeless. A national perspective, Nueva York, Plenum Press.

KOEGEL, P., A. BURMAN y S. MORTON (1996), "Enumerating Homeless People: Alternative Strategies and their Consequences", Evaluation Review, $\mathrm{n}^{\circ} 20$ (4), pp. 378-403.

LEONORI, L., M. MUÑOZ, C. VÁZQUEZ, J.J. VÁZQUEZ, M.F. BRAVO, M. NUCHE, P. BRANDT, A. BENTO y B. HORENBEK (2000), "The Mental Health and Social Exclusion European Network (M.H.S.E.): A Research Activity Report on European Homeless Citizens", European Psychologist, n5 (3), pp. 245- 251.

MARPSAT, M, (1995), Enquetes Pilotes Aupres des Sans-abri dans la Rue et Aupres des Utilisateurs de Services a Paris, Paris, INED.

MARPSAT, M. y J.M. FIRDIEU (1998), "Las personas sin hogar en París: Encuesta a una muestra representativa de usuarios de servicios para personas sin hogar", Intervención Psicosocial, $\mathrm{n}^{\circ}$ 7 (1), pp. 47-73.

MARTÍN, E. (1992), "Assessment of S-Night Street Enumeration in the 1990 Census", Evaluation Review, $\mathrm{n}^{\circ} 16(4), 418-438$.

MUÑOZ, M., P. KOEGEL, C. VÁZQUEZ, J. SANZ y A. BURNAM (2002), “An Empirical Comparison of Substance and Alcohol Dependence Patterns in the Homeless in Madrid (Spain) and Los Angeles (CA, U.S.)", Social Psychiatry and Psychiatric Epidemiology, n 37, pp. 289-298.

MUÑOZ, M. y C. VÁZQUEZ (1996), Estudio de los factores económicos y psicosociales que afectan a las personas sin hogar, Proyecto CICYT nº SEC96-1008-95, Plan Nacional I+D. 
MUÑOZ, M., C. VÁZQUEZ y J.A. CRUZADO (1995), Personas Sin Hogar en Madrid: Informe psicosocial y epidemiológico, Madrid, Comunidad de Madrid.

MUÑOZ, M., C. VÁZQUEZ, M. BERMEJO y J.J. VÁZQUEZ (1999), "Stressful Life Events among Homeless People: Quantity, Types, Timing, and Perceived Causality", Journal of Community Psychology, $\mathrm{n}^{\circ} 27$ (1), pp. 73-87.

MUÑOZ, M., C. VÁZQUEZ, P. KOEGEL, J. SANZ y M.A. BURNAM (1998), "Differential Patterns of Mental Disorders among the Homeless in Madrid (Spain) and Los Angeles (USA)", Social Psychiatry and Psychiatric Epidemiology, $\mathrm{n}^{\circ} 33$, pp. 514-520.

MUÑOZ, M., C. VÁZQUEZ y J.J. VÁZQUEZ (1998), Atrapados en la calle. Testimonios de personas sin hogar con problemas de salud mental, Madrid, Consejeria de Sanidad y Servicios Sociales de la Comunidad Autónoma de Madrid.

ROSSI, P.H. (1989), Down and Out in America, Chicago, University of Chicago Press.

STARK, R. (1992), "Counting the Homeless: An Assessment of S-Night in Phoenix", Evaluation Review, $\mathrm{n}^{\circ} 16$ (4), pp. 400-408.

VÁZQUEZ, C., MUÑOZ, M. y J. SANZ (1997), "Lifetime and 12-month Prevalence of DSM-III$\mathrm{R}$ mental Disorders among the Homeless in Madrid: A European Study using the CIDI", Acta Psychiatrica Scandinavica, ${ }^{\circ}$ 95, pp. 523-530.

VEGA, L.S. (1996), Salud Mental en Población Sin Hogar, Oviedo, SESPA.

WEISSMAN, M.J. y J. MARKOWITZ (1992), "Interpersonal Psychotherapy: Current Status", Archives of General Psychiatry, $\mathrm{n}^{\circ} 51$ (8), pp. 599-606.

WRIGHT, J. y J. DEVINE (1992), “Counting the Homeless: The Census Bureau's "S-Night" in Five U.S. cities”, Evaluation Review, n 16 (4), pp. 355-364. 
Anexo.

Cuestionario 1.

Plan Nacional I+D

Universidad Complutense

Enero 1997

ZONA / NOMBRE DE SERVICIO

ENTREVISTADOR (NOMBRE)

FECHA

Día mes año

$\mathrm{N}^{\circ}$ DE ENTREVISTA

\begin{tabular}{|c|c|c|c|c|c|c|}
\hline $\begin{array}{c}\text { Al. } \\
\text { Hora de contacto }\end{array}$ & $\begin{array}{l}\text { A2. } \\
\text { Género }\end{array}$ & Edad a & nada & $\begin{array}{c}\text { A4. } \\
\text { Procedencia }\end{array}$ & & A5 \\
\hline $\begin{array}{l}\mathrm{H}-{ }^{\prime}- \\
\operatorname{Min}-^{\prime}-\end{array}$ & $\begin{array}{cr}\mathrm{V} & 1 \\
& \\
\mathrm{M} & 2\end{array}$ & $\begin{array}{l}<20 \\
39-39 \\
59-59 \\
>60\end{array}$ & $\begin{array}{l}3 \\
4\end{array}$ & $\begin{array}{l}\text { España (no gitano) } \\
\text { España (gitano) } \\
\text { Magreb } \\
\text { Latinoamérica } \\
\text { África negra } \\
\text { Europa del este } \\
\text { Otros } \\
\text { Sin información }\end{array}$ & $\begin{array}{l}1 \\
2 \\
3 \\
4\end{array}$ & $\begin{array}{l}\text { Acepta } \\
\text { Rehusa } \rightarrow\end{array}$ \\
\hline $\begin{array}{l}\mathrm{H}-{ }^{\prime}- \\
\text { Min } \quad-^{\prime}-\end{array}$ & $\begin{array}{ll}\mathrm{V} & 1 \\
\mathrm{M} & 2\end{array}$ & $\begin{array}{l}<20 \\
39-40 \\
59-60 \\
>60\end{array}$ & $\begin{array}{l}1 \\
2 \\
3 \\
4\end{array}$ & $\begin{array}{l}\text { España (no gitano) } \\
\text { España (gitano) } \\
\text { Magreb } \\
\text { Latinoamérica } \\
\text { África negra } \\
\text { Europa del este } \\
\text { Otros } \\
\text { Sin información }\end{array}$ & $\begin{array}{l}5 \\
6 \\
7\end{array}$ & $\begin{array}{l}\text { Acepta } \\
\text { Rehusa } \rightarrow\end{array}$ \\
\hline $\begin{array}{l}\mathrm{H}-{ }^{\prime}- \\
\text { Min }-^{\prime}-\end{array}$ & $\begin{array}{cc}\mathrm{V} & 1 \\
\mathrm{M} & 2\end{array}$ & $\begin{array}{l}<20 \\
39-41 \\
59-61 \\
>60\end{array}$ & $\begin{array}{l}1 \\
2\end{array}$ & $\begin{array}{l}\text { España (no gitano) } \\
\text { España (gitano) } \\
\text { Magreb } \\
\text { Latinoamérica } \\
\text { África negra } \\
\text { Europa del este } \\
\text { Otros } \\
\text { Sin información }\end{array}$ & 9 & $\begin{array}{l}\text { Acepta } \\
\text { Rehusa } \rightarrow\end{array}$ \\
\hline
\end{tabular}




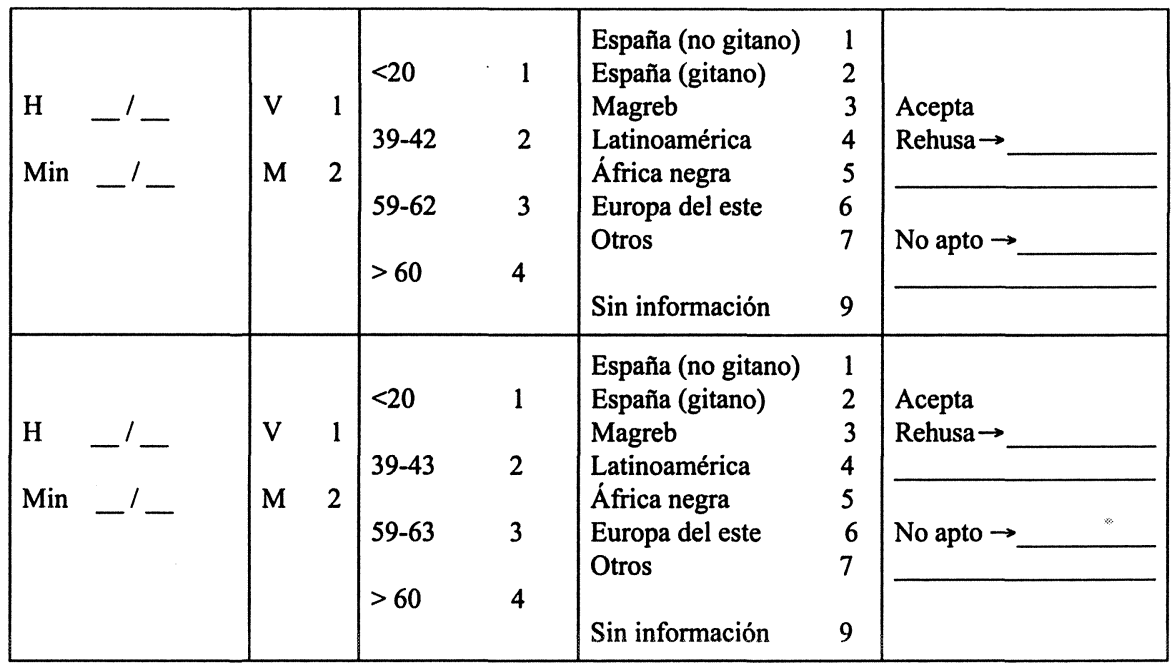

\section{PRESENTACIÓN RÁPIDA, EN FUNCIÓN DE LAS CIRCUNSTANCIAS.}

EJEMPLO: Hola. Buenas noches, perdone que le moleste. Estamos haciendo un estudio de la Universidad Complutense, me gustaría que me contestase a dos o tres preguntas muy rápidas.

C1. En las últimas cuatro semanas, ¿ha dormido al menos una noche en un albergue o centro de acogida?

$\begin{array}{ll}\text { Si (ESPECIFICAR) } & 1 \\ \text { No } & 2 \\ \text { Ns/Nc } & 9\end{array}$

ESPECIFICAR

C2. En las últimas cuatro semanas, ¿ha comido en algún comedor para personas sin hogar (aunque dependa de un albergue)?

$\begin{array}{ll}\text { Sí (ESPECIFICAR) } & 1 \\ \text { No } & 2 \\ \text { Ns/Nc } & 9\end{array}$

ESPECIFICAR 
RIS

REVISTA INTERNACIONAL DE SOCIOLOGIA

№ 32, Mayo-Agosto, 2002

M. MUÑOZ, C. VÁZQUEZ, J.J. VÁQUEZ, A.B. GUISADO y M. CRESPO

C3. En las últimas cuatro semanas, ¿ha utilizado alguno de los siguientes servicios?

$\begin{array}{llr}\text { ESPECIFICAR } & \text { BANOOS PÚBLICOS } & 01 \\ \text { ESPECIFICAR } & \text { ROPEROS } & 02 \\ \text { ESPECIFICAR } & \text { SERV. SANITARIOS } & 03 \\ \text { ESPECIFICAR } & \text { SERV. SALUD MENTAL } & 04 \\ \text { ESPECIFICAR } & \text { UMES } & 05 \\ \text { ESPECIFICAR } & \text { CEDIA } & 06 \\ \text { ESPECIFICAR } & \text { PARROQUIAS } & 07 \\ \text { ESPECIFICAR } & \text { OTROS ALOJAMIENTOS } & 08 \\ \text { ESPECIFICAR } & 09 \\ & \text { OTROS SERVICIOS } & 99\end{array}$

